\title{
Le soufisme New Age des salles de yoga *
}

\author{
DALIA CHAMS
}

\begin{abstract}
In trendy yoga studios, a new type of Sufism practice is flourishing in Egypt, that we can call New Age Sufism. It's merging traditional Sufi practices as $\underline{d} i k r$, with Zen meditation and yoga breathing techniques. People who attend this kind of sessions are more or less highly ranked; they are looking for another way to reach serenity and inner peace, to avoid post-revolution stress.

This paper aims to analyse this kind of practice by first describing a Sufi meditation session, in order to explain how it creates a new type of Sufism New Age, more universal and not linked to a religion in particular. Then, it sheds light on the target group this neo-Sufism is attracting and the reasons behind these practises. At the end, the paper emphasises other related aspects of this phenomenon in the cultural field.
\end{abstract}

Keywords: Sufism, spirituality, yoga, Zen, New Age

\section{Introduction}

Des lumières tamisées, des encens, des bougies,... l'ambiance des studios de yoga se prêtent bien à recevoir toute sorte d'idées Zen. Influencées par des écoles et des confessions différentes, elles communient harmonieusement ensemble. On médite en ayant recours aux noms d'Allah, on pratique des nouvelles formes de $\underline{d} i k r$-tendance, un peu différent des séances traditionnelles où l'on psalmodie les noms de Dieu, dans une sorte de danse sacrée.

En fait, ce genre de studios a de plus en plus le vent en poupe, notamment ces sept dernières années. Les activités qu'ils organisent, annoncées sur Facebook ou autres sites Web, allant des cours de soufisme musulman, aux classes de yoga, en passant par l'ésotérisme pharaonique ou le Taï Chi, le Reiki, la méditation, les retraites et c... ciblent surtout des personnes qui cherchent à atteindre la sérénité et le rapprochement des

This paper is describing a new social practice in Egypt that occurred after the revolution of 25 January 2011. People are trying to find a new way to connect with Allah and to deliver their pains, by merging Sufism and new age rituals. It has been conducted in the framework of the In 2016 project and the corresponding publications "Living 2016" in JAIS 16 (2016), and "Arrays of Egyptian and Tunisian Everyday Worlds" in JAIS 17 (2017). 
Hommes. Elles sont en quête d'autres voies spirituelles, parsemées des notions de «Dieu » et de «l'amour », en dehors du cadre traditionnel de la religion comme prêchée par les plus rigoristes. Pour échapper à un quotidien de plus en plus lourd à assumer, elles établissent un parallèle entre l'extinction (le $\operatorname{fan} \vec{a}$ ' soufi) et le nirvana. Et sont prêtes à payer des tarifs onéreux, à l'entrée, ou de faire volontairement partie des clients du «supermarché » spirituel, en vogue partout dans le monde.

Au lendemain de la révolution de 2011, ensuite avec l'accession des Frères Musulmans au pouvoir, plusieurs ont recours au coaching, à l'aide des spécialistes de développement humain ou carrément aux psychologues et psychiatres, afin de les aider à surmonter les pressions et les difficultés de la vie. On a surtout moins honte de demander une aide psychologique, car la révolution a fait aussi tomber plein de tabous sociaux. Certains ont choisi de se tourner vers Allah différemment ; ils encouragent l'introspection et réalisent que Dieu est à l'intérieur de soi-même.

Les idées du poète soufi Ğalāl al-Dīn Rūmī peuvent être ainsi considérées comme «mahométines Zen»; le grand public égyptien est en train d'ailleurs de les redécouvrir, grâce notamment au succès indélébile du roman de l'écrivaine turque, Elif Shafak, Soufi Mon Amour (Qawāid al-'išq al-arbacūn, traduit vers l'arabe en 2013). Depuis sa sortie, l'ouvrage ne cesse d'être sur la liste des best Sellers et fait office d'un livre de chevet pour pas mal de jeunes. Ceux-ci postent de temps en temps des extraits du roman sur Facebook, les citations entières sont reprises en chœur par les amis, pour affirmer que ce genre d'amour peut effectivement changer leur existence et que le livre a constitué un tournant dans leur vie. L'engouement se poursuit et revêt une autre forme spirituelle, à savoir les concerts de musique soufie, les cercles de $\underline{d} i k r$ contemporains, qui se déroulent parfois à hui clos dans les studios de yoga (comme celui décrit plus haut) ou dans des vieilles maisons restaurées, transformées par des particuliers en des centres culturels «indépendants ». A titre d'exemple, Bayt Ḥusn (à la rue Umm al-Ġulām) et al-Raba' (à la rue al-Mu'izz) accueillent régulièrement ce genre de soirées musicales, dès leur ouverture assez récente. De nouvelles troupes et interprètes puisent dans le patrimoine ancien, se permettent parfois des mélanges intéressants et se présentent dans ces nouveaux espaces culturels. Facebook leur sert de véritable support médiatique et ils finissent par se trouver sur les podiums.

Le papier que nous proposons doit faire l'état des lieux sur ce soufisme New Age, si l'on ose dire, analyser ses divers aspects (pratiques sociales, musicales ou littéraires) et son rapport à la révolution, essentiellement selon une démarche empirique et une observation participative. Ce, dans le but de mieux cerner l'évolution des mentalités, des désirs et des attentes d'une tranche de la société égyptienne.

Dans un premier temps, nous décrivons la démarche suivie lors d'une séance de méditation soufie, afin d'expliquer le principe de ce soufisme New Age et comment il fait office d'un culte universel, non lié à une religion en particulier.

Ensuite, nous abordons en détails les principales caractéristiques du public ciblé (le terreau sociologique). Puis, nous nous attaquons aux raisons qui justifient le recours au soufisme Zen. Et enfin, nous concluons sur d'autres aspects littéraires et artistiques en lien avec ce genre de pratiques. 


\section{Le culte de l'universel}

Elle s'appelle Sonia. Séjourne tous les ans à peu près quatre mois en Égypte, entre décembre et mars, pour échapper au froid des Etats-Unis dont elle porte la nationalité. De père et mère égyptiens, elle a passé la majorité de sa vie à Chicago. Mais a décidé, depuis la révolution de 2011, de venir plus souvent dans son pays d'origine, pour tenir des séances de méditation soufie. C'est d'ailleurs son activité professionnelle qu'elle pratique aux USA, où elle a suivi des études de trois ans, à l'University of Spiritual Healing and Sufism, fondé par son mentor spirituel, cheikh Muḥammad al-Ğamāl, qui s'est éteint en novembre 2015.

Mon père est mort et nous sommes venus l'enterrer en Égypte, juste avant la révolution. Quand j'ai décidé d'aider les gens en pratiquant la méditation soufie, en Égypte, je ne connaissais personne, mis à part 3 ou 4 amis et la famille. J'ai fait une prière et Dieu m'a aidée à rencontrer les gens qu'il fallait, lesquels étaient prêts à m'accueillir dans leurs studios de yoga ou cafés. Je ne savais pas qu'elle serait la réaction du public, car j'étais la première en Égypte à mélanger le soufisme et la méditation Zen. Pourtant, aux Etats-Unis, je reçois souvent des non musulmans, qui ne comprennent pas la signification des divers noms de Dieu que j'emploie. Il est évident qu'il y a quelque chose qui pèse davantage sur les gens en Égypte. Plus de pressions, notamment dans le contexte postrévolutionnaire. En outre, la population est très nombreuse, donc on s'expose à beaucoup d'énergies, parfois contraires, et beaucoup d'émotions. Ceci affecte tout le monde, ce n'est pas évident d'y échapper, ${ }^{1}$

dit-elle, en ajustant le foulard lui couvrant la tête, pour mieux préserver son champ énergétique. Il glisse légèrement, laissant paraître ses longs cheveux tressés.

Sonia Hasan, 45 ans, se recueille dans la salle de méditation, seule, se prêtant à un rituel à même de la «nettoyer de la poussière du monde ». Elle s'apprête ainsi à recevoir les nouveaux venus, comme les fidèles qui attendent son retour annuel, afin de profiter des séances de méditation, qu'elle offre et où elle se sert des noms de Dieu comme mantras. Mêlant les techniques de respiration du yoga, à celles de la méditation syncrétique visant à recharger les centres d'énergie dans le corps ou chakras, elle répète un nom spécifique de Dieu lequel correspond à un organe en particulier. Et ce, après avoir récité la fātiha, la première sourate du Coran, et égrené plusieurs fois le mot Allāh, en détachant les syllabes, suivant le rythme de la respiration.

Jeunes et moins jeunes s'agglutinent autour de leur coach, dans la salle de méditation, à peine éclairée par une lampe couverte d'habits de soie multicolore. Assis en tailleur, sous la forme d'un demi-cercle, ils constituent un espace interconfessionnel pour toute l'humanité. La règle est donc de n'interférer avec l'idéal de personne, mais de célébrer les idéaux de tous. L'odeur des bougies parfumées et de l'encens indien emplisse l'atmosphère de ce studio cairote de yoga à la mode.

Sonia Hasan demande à la présence, essentiellement en anglais, en incrustant quelques mots d'arabe, de s'introduire très brièvement à tour de rôle et de mentionner la raison pour laquelle ils ont pris part à ce cercle. Certains se livrent à un témoignage aux accents sincères sur leur trajectoire et leur expérience émotionnelle avec la méditation, comme dans

1 Entretien avec Sonia Ḥasan, coach de soufisme et guérison spirituelle, le Caire, mars 2018. 
un petit groupe de soutien ou de thérapie. Une jeune femme est en pleurs, peut-être par apitoiement sur soi-même.

La coache spirituelle ne manque pas au début de chaque séance de montrer le nom d'Allah, en calligraphie arabe, et de raconter l'histoire de son père, médecin, mort juste avant la révolution de 2011. Dans ses papiers, elle a trouvé le dessin d'un cœur humain, où il a inscrit dans chacune de ses quatre chambres une lettre du mot Allāh en arabe, dit-elle, avant d'égrener le nom de Dieu, l'unique, l'univers, al-ḥaqq (l'absolu ou la vérité) et de demander à tout un chacun de faire un vœu silencieusement. Dans l'esprit, il faut par la méditation atteindre le vide mental ou le silence des pensées, pour entrer, après, dans la claire lumière ou lâcher prise. Et pour ce faire, il faut "polir le miroir de son cœur», comme dirait le grand poète mystique Ğalāl al-Dīn Rūmī :

On recherche mentalement le chemin vers lui. Cela ne marche pas, Mais le moment où épuisé, on lâche, Il n'y a plus d'obstacle. Il se présente lui-même à nous...Lorsque vous lâchez prise, Dieu est présent. Comment autrement aurionsnous pu le connaître?

Le mouvement soufi est un élément important des courants de pensées liées aux religions du cœur. Pour ce, Sonia Hasan débute sa méditation par une respiration et une expiration lentes, en focalisant sur le cœur, le remplissant des vibrations du son Allah, tout en plaçant sa main droite, à gauche, sur le cœur. Le son de sa voix a une riche résonnance.

Je me réfère à la science de la BioGéométrie, développée par Ibrāhīm Karīm [un architecte et scientifique égyptien né en 1942]. Celle-ci traite de la vibration de l'énergie de la forme, de la couleur et du son, dans le but d'harmoniser l'équilibre dans les champs d'énergie. Nos émotions sont énergie, nos pensées sont énergie, notre vitalité est énergie. Tous types de formes, de sons ou de couleurs affectent ces niveaux d'énergie. J'utilise, ainsi que d'autres coachs spirituels les noms de Dieu indiqués par Karim, allant de pair avec chacun des organes », ${ }^{2}$

résume Sonia Hasan.

Son discours est parsemé des notions de «Dieu» et «amour», ainsi que d'idéal d'universalité fraternelle, harmonisant toutes les religions. De quoi répondre aux besoins des personnes qui cherchent à atteindre la sérénité et le rapprochement des Hommes, grâce à l'intelligence de la raison et du cœur. Celles qui sont en quête d'autres voies spirituelles, en dehors du cadre traditionnel de la religion comme prêchée par les plus rigoristes.

Cette transformation du soufisme en un uni-diversalisme capable de répondre à toutes les aspirations spirituelles, n'est-elle pas symptomatique des logiques de la modernité religieuse ? (...) Ne génère-t-elle pas alors quelques fois des demandes religieuses que l'offre locale ne satisfait plus, ou plus entièrement ? Les acteurs se retournent alors vers des ressources symboliques et institutionnelles en Occident comme Sufi Order International, et réinventent leur religiosité selon les dynamiques du croire contemporain,

2 Entretien avec Sonia Ḥasan, coach de soufisme et guérison spirituelle, le Caire, mars 2018. 
souligne la chercheuse Alix Philippon, en juillet 2014, dans son papier académique intitulé De l'occidentalisation du soufisme à la réislamisation du New Age?

\section{Le Zen mahométin}

Sufi Order International (SOI) a été fondé au début du XX ${ }^{\text {ème }}$ siècle en Occident et pour un public occidental par Hazrat Inayat Khan (1916-2004), un musicien et disciple de l'ordre soufi indien de la Chishtiyya. Ce groupe offre l'exemple d'un soufisme occidental ayant trouvé sa place dans la nébuleuse New Age, en embrassant des formes syncrétiques de spiritualité et en mettant l'accent sur l'universalisme du message soufi, par delà l'islam.

Ainsi, ce soufisme a réussi à se déterritorialiser, sous l'effet de la globalisation religieuse. De quoi avoir engendré à la fois sa «dé-islamisation» et sa «stylisation », lui permettant de trouver sa place dans le marché religieux actuel, de par le monde, plus précisément dans la nébuleuse mystique ésotérique.

Les thèmes ésotériques développés par ce soufisme présentent des continuités évidentes avec les mystiques orientales: l'idée d'un divin immanent, la focalisation sur la transformation intérieure ou encore la dimension émotionnelle de cette mouvance religieuse ne présentent pas de ruptures essentielles avec l'univers classique du soufisme. Ce qui est nouveau, c'est que le soufisme New Age soit « désorbité » de l'unique référence islamique et qu'il se revendique comme voie initiatique, hors de toute orthodoxie. La preuve est que dans les salles de méditation cairote, l'on retrouve souvent des non musulmans étrangers, qui parlent très peu l'arabe, mais qui tiennent à être présents, correspondant parfois avec les coachs par Internet, avant de venir les voir. De même, plusieurs femmes de confession musulmane, voilées et non voilées, précisent que ce genre de méditation les réconforte dans leur doctrine.

$\mathrm{Au}$ lieu de recourir à des mantras en sanskrit dont on ignore le sens, recourir aux noms de Dieu ne peut être qu'une bénédiction, notamment que l'effet est presque le même, si ce n'est plus fort, ${ }^{3}$

avoue 'Azza, une femme dans la quarantaine, laquelle a souvent fréquenté les cercles de yoga et de séances ésotériques, par curiosité, mais aussi par besoin de calmer l'esprit et le cœur. D'ailleurs, elle n'a pas manqué d'y emmener son propre fils, étudiant en polytechnique, pour l'aider à recouvrer le chemin vers la paix intérieure. Car parfois, le recours aux biens spirituels prolonge des thérapies psychologiques ou psychanalytiques, ou accompagne une sorte de bricolage spirituel, allant du yoga, au Taï chi, au Reiki etc. La même « clientèle » apprécie souvent les idées de Ğalāl al-Dīn Rūmī ou des poètes indien et pakistanais, Kabīr et Muḥammad Iqbāl, considérées comme «mahométines Zen ».

Mais en se vulgarisant, le soufisme New Age risque de décomposer progressivement son message essentiel et de basculer dans la dérive syncrétique, avancent les uns. Si certains courants dits soufis acceptent de ne plus le lier intrinsèquement à l'islam ou d'intégrer au soufisme des éléments extérieurs, pour faire des émules, ils risquent de le

3 Témoignage recueilli au Caire, janvier 2018. 
transformer en un genre de mode, une tendance. Celle-ci favorise la construction de petites mythologies personnelles, agrégats de croyances diverses et de pratiques plus au moins « relaxantes » à consonance orientale. C'est plutôt dans ce cas-là un goût qu'on suit «en électron libre» et non plus un engagement personnel profond, loin des voies soufies authentiques.

Evidemment, les populations lassées et stressées par le rythme infernal de la vie peuvent embrasser à bras ouverts ces modes de vie New Age qui leur apportent la réponse à leurs besoins, comme l'ont fait les Américains dans les années 1960 qui cherchaient à instaurer un nouveau paradigme de vie. On assiste en Égypte à un phénomène qui peut-être comparer aux sixties américains, ouvrant la voie à une foule de psychologues, formateurs, coachs, thérapeutes, conseillers en ressources humaines.

Ces acteurs nouveaux se sont présentés comme des spécialistes du développement personnel. Sous leur influence, d'innombrables techniques ont vu le jour. Des stages, des séminaires, des consultations ont attiré un public de plus en plus large. Les manuels de développement personnel ont inondé les rayons des libraires. Un véritable marché de l'épanouissement s'est constitué,

indique Michel La Croix, dans son livre Se réaliser, Petite philosophie de l'épanouissement personnel. ${ }^{4}$

Le développement personnel fait désormais office de phénomène de mode, en Égypte. Il annonce la possibilité d'entamer une redécouverte du sacré, tout en insistant sur l'importance de la dimension spirituelle de l'homme, rejetant une vision purement rationaliste et matérialiste de l'humanité, notamment au sein d'une société encline à des changements fulgurants et à l'ombre d'une vie qui devient de plus en plus dure.

Dans cette optique, la culture New Age s'impose de par la volonté d'un futur meilleur. (Le New Age englobe un grand nombre de religions qui diffèrent dans les pratiques selon les vocations, les sensibilités et les cultures, mais qui somme toute délivre un même mode de pensée, basé sur les enseignements théosophiques de Helena Blavatsky: kabbale, Bouddhisme, Krishna, chamanisme,... Certains penchent à le décrire comme le nouvel ordre mondial, sous l'habit de la spiritualité).

Loin d'être un réseau unifié et uniforme, le New Age est au contraire un réseau fluide d'adeptes dont l'approche est de "penser globalement et d'agir localement». Cette expression du psychiatre suisse, Carl Gustave Jung, résume une façon de penser qui peut mener à une société alternative et holistique, dans une frénésie de révolte contre une autre extrêmement consumériste. L'alternative envisagée est donc une transformation de soi pour transformer le monde, et ainsi permettre une articulation harmonieuse de l'un et du multiple. Ceci dit transformer l'individu pour changer le collectif par un effet de contagion, comme le prône Sonia Ḥasan et d'autres life coachs, dans leurs séances : commencer par soi-même, et tout le reste viendra. Seul l'homme riche intérieurement et extérieurement peut être heureux et symboliser un espoir pour un changement sur cette planète.

4 LA CROIX 2009: 158.

ز 18 (2018): 51-62 


\section{Le terreau sociologique}

Si la séance, d'environ l'heure et demie, coûte au moins 100 ou $200 £ E$ elle répond surtout aux demandes d'une bourgeoisie libérale et occidentalisée, réfractaires aux offres religieuses généralement disponibles sur place. Celle-ci semble avoir perdu foie en ceux qui se proclamaient d'un islam modéré, tels les Frères Musulmans une fois au pouvoir, ou même certains prédicateurs médiatisés qui défrayaient la chronique avant la révolution de janvier 2011. Sur ce, le soufisme New Age des salles de yoga peut s'inscrire dans le cadre de nombreuses tentatives de production de la modernité à partir de la tradition. La mondialisation n'a pas uniquement un caractère économique ou géopolitique, mais elle a un versant plus discret quant aux échanges accrus sur le plan interreligieux. (Il y a quelques années le prédicateur à la mode, 'Amr Hālid, avait puisé son discours et la mise en scène de ses émissions télévisées dans la tradition évangéliste). Comme l’assure Alix Philippon,

la globalisation du religieux se déploie selon des configurations déterminées par la demande d'acteurs sociaux, en quête d'identité, de promotion culturelle et sociale, de restructuration personnelle ou encore de méthodes alternatives de santé et de mieux être (...) Ce qui fait l'unité de cette mouvance est que c'est une religiosité éclectique centrée sur l'individu. ${ }^{5}$

Ces groupes ne correspondent ni au type de l'église ou de la mosquée, ni à celui de la secte, mais davantage à celui du «groupe flottant» de type mystique proposé par le théologien Ernest Troeltsch, caractérisé par l'expérience personnelle immédiate à distance des formes objectivées de croyances et de cultes par une association très lâche, qui privilégie les liens personnels par affinité spirituelle.

On vient y chercher un service spirituel ponctuel, un bien être, une expérience émotionnelle collective, mais on vient librement et on sort librement.

Pour l'adepte, la fonction première de l'engagement dans ce groupe est de lui permettre de déployer ses potentialités dans l'arène du quotidien en vue de gagner en sérénité, en cohérence intime, et à des fins utilitaires, d'obtenir des résultats et des succès mondains,

affirme Alix Philippon ${ }^{6}$ dans le même papier.

Les participants à ces séances sont largement issus de la petite bourgeoisie nouvelle, au capital culturel élevé (travailleurs sociaux, enseignants, professions médicales, artistiques et culturelles). C'est dans ce terreau sociologique que se déploient réconciliés, les désirs d'autonomie individuelle et d'ancrage qu'offrent les nouvelles formes de communisation religieuse. L'acquisition de ces nouveaux savoirs ésotériques compenserait, en termes de valorisation de soi, une certaine frustration engendrée par le décalage entre ce capital culturel et un statut social perçu parfois comme peu gratifiant, le fait remarquer Philippon. Plus encore, ces cercles sont souvent propices aux rencontres, amicales ou amoureuses ; elles servent à créer un lien social entre semblables.

5 PHILIPPON 2014.

6 Ibid. 
Ce soufisme occidentalisé peut être considéré aussi comme une matrice de réislamisation pour des acteurs de la bourgeoisie cosmopolite et libérale urbaine. Leurs pratiques et leurs discours manifestent des dynamiques de réinvention de la subjectivité dans le cadre d'une religiosité non autoritaire, notamment dans son rapport au maître soufi, et qui affirme dans sa doctrine la centralité de l'individu, de l'humanisme et du dialogue.

\section{Pourquoi maintenant?}

Ces acteurs sont en accord avec l'idéologie anti-islamiste du pouvoir. Et ce, dans le cadre de la guerre contre le terrorisme qui bat son plein en Égypte comme dans plusieurs pays du monde arabo-musulman. D'autant plus, que les radicaux s'attaquent souvent aux sites et aux adeptes soufis, les jugeant peu orthodoxes.

Il y a inéluctablement une classe, dotée d'un certain bagage intellectuel, qui sans être laïque, aspire à se ranger sous un étendard souple ou à adopter une solution à l'intolérance ambiante et aux déclarations belliqueuses de tous ces autres groupes pensant détenir le monopole de la vérité religieuse.

Sonia Hasan, elle-même, est issue de l'immigration, comme pas mal de jeunes gens. Elle était à la recherche de la vérité. Et après avoir tenté plusieurs chemins, allant des études artistiques aux cours de massage, elle a été attirée par l'islam plus spirituel, moins juridique, comme le lui a présenté son mentor cheikh Muḥammad al-Ğamāl al-Rifāc̄̄ alŠădilīi. Descendant du cheikh Aḥmad Al-Rifāē̄i, il ne se contentait pas de lui indiquer ce qui est permis et interdit, mais allait beaucoup plus loin dans ses instructions. Né en 1935, à Toulkarem en Cisjordanie, il a vécu à Bethléem et à Jérusalem où il a travaillé entre autres en tant que juge, réputé pour sa sagesse et son savoir faire. (Avant de s'installer aux EtatsUnis et de fonder l'University of Spiritual Healing, ayant des succursales au Canada, en Mexique et en Argentine).

Le cadre souple de ce soufisme moderne ressemble peu aux règles et à l'hiérarchie conventionnelle, imposées aux fidèles dans les milieux populaires ou pauvres, et au sein des confréries islamistes. Cet état des lieux s'applique à l'Égypte, mais aussi au Maroc, au Pakistan, aux Emirats arabes unis, comme le soulignent quelques écrits publiés en arabe à ce sujet. Par exemple au Maroc, le roi Muḥammad VI a adopté une politique visant à redessiner le paysage religieux, invoquant essentiellement le soufisme et a nommé, à la tête du ministère des Waqfs (biens de main morte) le cheikh Aḥmad Tawfīq lequel appartient à ce courant, traditionnellement encouragé par la monarchie.

Il n'y a pas un mois qui se déroule sans la tenue d'une manifestation soufie, sous le parrainage de la royauté (...) Et parmi la classe intellectuelle francophone, c'est surtout un nouveau soufisme franco-américain qui a le vent en poupe,

mentionne un long article publié par Nawfal Bin Ibrāhīm. ${ }^{7}$

De même, au Pakistan, le soufisme a été réinterprété comme un islam, « anti-mollahs » et a servi d'outil à la «modération éclairée » du général Pervez Musharraf qui visait, entre

7 BIN IBRĀHĪM [s.d.]

ز 18 (2018): 51-62 
autre, à promouvoir une version tempéré de l'islam et une image moderne du pays. C'est également le cas aux Emirats arabes unis, à quelques variations près, sous la houlette du cheikh Muhammad Bin Zāyid qui œuvre depuis 10 ans à intégrer le soufisme à l'identité nationale des émirats.

L'Égypte ne fait pas exception à cette règle qui semble dominer la région. Comptant quelque 80 confréries soufies, regroupant environ 9 millions d'adeptes, ces confréries ont scandé en juin 2015 le slogan : « le soufisme contre l'extrémisme », en réponse à l'appel du président 'Abd al-Fattāḥ al-Sīsī visant à réformer le discours religieux. Ce dernier appartient à l'ordre soufi des Ğa'fariyya, dit-on dans les coulisses, afin de justifier les termes soufis récurrents dans ses interventions orales. Aḥmad al-Ṭayyib, le grand cheikh d'al-Azhar, est lui aussi un soufi de Louqsor bien évidemment. Et l'ancien muftī, le cheikh 'Alī Ğum'a, assez proche des instances du pouvoir, affiche également son appartenance soufie. Plus encore, il a obtenu une autorisation officielle, en février 2018, afin de fonder une nouvelle confrérie soufie dont il sera le chef spirituel, à savoir al-Šādiliyya al-'Āliya, qui dès sa création, a pu s'attirer 400 mille adeptes à l'intérieur et à l'extérieur du pays.

Toutes ces figures de proue de la société égyptienne parie sur la capacité du soufisme à rééquilibrer la vie religieuse, sur le plan local. Il s'agit en effet d'un son de cloche que l'on entend également en Occident, par les spécialistes et observateurs, depuis le 11 septembre 2001.

Les ordres soufis possèdent une véritable force d'attraction. Les gens y trouvent une forme de religion qui les conforte et allège les fardeaux de la vie, notamment qu'ils ne font pas de distinction entre leurs membres selon des critères socioéconomiques. (...) Répandus aux quatre coins de l'Égypte, l'Etat centralisé cherchait à avoir mainmise sur eux, afin d'assurer un meilleur contrôle des classes et des territoires. Il ne les regardait pas d'un mauvais œil, étant donné qu'ils prêchent un message pacifique [de résignation et de repentir-D.Ch.] poussant à mieux accepter la fatalité. De quoi ne constituer aucun danger pour le régime en place,

explique Hiba Šarīf, dans son ouvrage Dīnī wa-dīn al-nās (Ma religion et celle des autres). ${ }^{8}$

Cette pratique religieuse soft écarte la colère et l'insurrection, tout en permettant aux citoyens de revenir vers Dieu, dans les moments difficiles, réclamant sa justice et sa tolérance. Ils en ont grand besoin, notamment à l'issue des printemps arabes et les frustrations qui les ont suivies.

Cet état de fait arrange parfaitement les Egyptiens, ceux des couches populaires qui fréquentent les cercles de $\underline{d} i k r$ traditionnels, où l'on égrènent les noms de Dieu, comme ceux de la classe bourgeoise qui se réunissent dans les salles de yoga pour exercer la méditation, toujours en égrenant les noms de Dieu, suivant les rythmes de la respiration et les vibrations sonores.

Les réseaux sociaux ne font qu'accroitre l'effet de ces idées de tendance soufie, en permettant un plus grand échange entre adeptes, mais aussi en favorisant le contact entre adeptes et coach de soufisme et guérison spirituelle, à l'instar de Sonia Hasan, qui a lancé une branche de Sufi Spiritual Healing en Égypte, à travers une page Facebook qui porte le

8 ŠARĪF 2017: 136-138. 
même titre. Celle-ci sert à créer un réseau, à annoncer les évènements relatifs à son objectif. De même, tous les studios accueillant souvent ce genre d'activités tels Mudra, Shanti, Rouh, Sufi café etc. réactualisent régulièrement leurs pages Facebook, s'attirant davantage des curieux qui viennent satisfaire une spiritualité mal comblée.

J'ai découvert Sonia Ḥasan, en suivant les annonces de studio Shanti, sur Facebook et What's up, comme j'ai suivi avant les cours de Madame Sanā' al-Ğabūrī, une spécialiste irakienne du soufisme, adepte de la confrérie al-Qādiriyya, qui suivait aussi un gourou basé à Kuala Lumpur en Malaisie. Celle-ci, de style plus conventionnel, rapprochait également la BioGéométrie, développée par Ibrāhīm Karīm, évoquant la vibration de l'énergie du son, de la forme et de la couleur. Elle précisait par exemple, à quel moment de la journée les vœux et les prières sont plus exaucés, en fonction des heures et du rapport à la nature. Et expliquait comment se protéger des ondes négatives, à l'aide de textes coraniques,

raconte l'une des femmes qui fréquente le studio. ${ }^{9}$

Outre les séances de méditations soufies, de reiki et autres, les réseaux sociaux de ces salles de yoga annoncent également des concerts ou spectacles, liés à la même thématique. Du coup, on peut y découvrir sur certains parmi eux les chants d'un ensemble marocain comme Ibn 'Arabī ou syrien comme al-Riḍ̂ān (les fils de Mar'ašlī) qui se produisent en Égypte, avec succès.

\section{La veine de Soufi mon amour}

Un chant soufi, une belle mélodie transcendante peut attirer vers une méditation soufie et vice versa. Roumi ne rappelle-t-il pas constamment que la méditation ne peut se séparer de l'ouverture du cœur et que la vie est une célébration, une danse ?

Une chose est sûre, le public de ce genre de concerts, connaissant souvent le répertoire des troupes par cœur, est presque le même que celui des salles de yoga. Des vieilles maisons aménagées en centres culturels comme Bayt H̦usn et al-Raba dans le vieux Caire fatimide (voir ci-dessus, p. 52) accueillent régulièrement ce genre de soirées musicales à succès.

Par contre, rien ne peut égaler le succès foudroyant du roman de l'écrivaine turque, Elif Shafak, Soufi Mon Amour (traduit vers l'arabe en 2013 sous le titre Qawä'id al-iš́q alarba'ün). Les responsables de la librairie Tanmiya, au centre-ville cairote, lesquels avait le monopole de sa distribution en Égypte, affirment avoir vendu plus de 10 millions d'exemplaires à sa sortie, ajoutant que l'ouvrage continue à figurer sur les listes des bestsellers, au bout de cinq ans de sa parution. Et ce, sans compter le nombre de copies fraudées qui se vendaient sur les trottoirs à des prix dérisoires. Un tel engouement trouve probablement sa raison en un état de fatigue générale et une soif spirituelle que les paroles de Šams de Tabrīz, l'un des principaux personnages du roman étanche en disant :

9 Témoignage recueilli au Caire, en l'été 2016. 
Rien ne doit vous séparer de Dieu. Personne ne doit servir d'intermédiaire ni des imams, ni des prêtres, ni des rabbins. Il n'y a pas de tuteur moral ou religieux, ni de seigneurs spirituels.

Ce roman, qui retrace la rencontre entre le poète Roumi et le plus célèbre derviche du monde musulman, Šams de Tabrīz, est une révélation pour Ella Rubinstein, à l'aube de ses quarante ans. Le texte va chambouler son existence, comme il l'a fait avec les lecteurs, découvrant au fil des pages, le soufisme, le refus des conventions et la splendeur de l'amour.

L'écrivain 'Alā' al-Dīb, dans une analyse du livre, à sa sortie, explique qu'en lisant, on arrive au constat suivant :

Le $21^{\text {ème }}$ siècle et le $13^{\text {ème }}$, cadre temporel de l'histoire, se ressemble pour beaucoup. Ils ont connu les mêmes guerres religieuses inégalées, la même mésentente culturelle et le même sentiment de peur et d'insécurité. ${ }^{10}$

Le roman porte la réponse, il faut se dire: l'amour est ma religion et ma foi. Elif Shafak écrit aussi :

Nous ne devons oublier que la bougie nous aide juste à nous déplacer dans l'obscurité. Quel intérêt d'être très concentré sur la bougie, au point d'oublier notre destination?

La voix de Sonia Hasan vient de si loin : «Il faut rester connectés, sans se perdre dans le détails $»$. L'encens était complètement consumé. Il ne restait pas la moindre trace de son odeur dans la salle.

\section{Bibliographie}

ANONYME. [s.d.]. «La BioGéométrie (BioGeometry), une science ancestrale développée par le Dr Ibrahim Karim ». <https://de-coeur-a-coeur.net/produits-biogeometrie/> (consulté le $1^{\text {er }}$ avril 2018).

ANONYME. [s.d.]. «Les origines modernes du développement personnel ». 〈http://kpsens.com/lesorigines-modernes-du-developpement-personnel/> (consulté le $1^{\text {er }}$ avril 2018).

BIN IBRĀHĪM, Nawfal. [s.d.]. «al-Tașawwuf al-frankū-amīrikī al-ğadīd fì 1-Mağrib » (Le nouveau soufisme franco-américain au Maroc). 〈http://www.saaid.net/feraq/sufyah/104.htm?print_it=1> (la consulté le $1^{\text {er }}$ avril 2018).

al-DīB, 'Alä’. 2013. «Al-ğanna al-ān wa-kad̄ālik al-ğaḥimm » (Le paradis maintenant, mais aussi l'enfer). Al-Mașrī al-Yawm, 11 mai 2013. <https://dbonfrdgauzmg.cloudfront.net/news/details/ 196964> (consulté le $1^{\mathrm{er}}$ avril 2018...).

IDRISS, Chah. [2015]. Al-soufiyoune / traduit de l'anglais vers l'arabe par Bayoumi Qandil. Centre national de la traduction, Le Caire.

10 al-Dīв 2013. 
Julien, B. 2010. «Les tentations du soufisme moderne ». 15 septembre. <http://www.1001nuits.org/ index.php?title=Les_tentations_du_soufisme_\%22moderne\%22> (consulté le $1^{\mathrm{er}}$ avril 2018.).

La Croix, Michel. 2009. Se réaliser : Petite philosophie de l'épanouissement personnel. Édition Robert Laffont, Paris.

PhiLipPON, Alix. 2014. «De l'occidentalisation du soufisme à la réislamisation du New Age ? Sufi Order International et la globalisation du religieux ». Revue des mondes musulmans et de la Méditerranée, 135: 209-226. 〈https://journals.openedition.org/remmm/8487>.

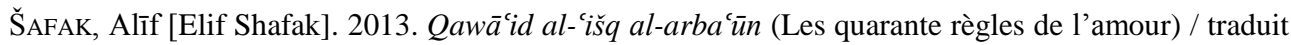
de l'anglais vers l'arabe par Muḥammad Darwīšs. Dār al-Ādāb, Beyrouth.

ŠARĪF, Hiba. 2017. Dīn̄i wa-dīn al-nās (Ma religion et celle des autres). Éditions al-'Arabī. 